\title{
Global Existence and Convergence of Solutions to a Cross-Diffusion Cubic Predator-Prey System with Stage Structure for the Prey
}

\author{
Huaihuo Cao ${ }^{1}$ and Shengmao Fu ${ }^{2}$ \\ ${ }^{1}$ Department of Mathematics and Computer Science, Chizhou College, Chizhou 247000, China \\ ${ }^{2}$ College of Mathematics and Information Science, Northwest Normal University, Lanzhou 730070, China
}

Correspondence should be addressed to Huaihuo Cao, caguhh@yahoo.com.cn

Received 3 December 2009; Accepted 30 March 2010

Academic Editor: Wenming Zou

Copyright (C) 2010 H. Cao and S. Fu. This is an open access article distributed under the Creative Commons Attribution License, which permits unrestricted use, distribution, and reproduction in any medium, provided the original work is properly cited.

We study a cubic predator-prey system with stage structure for the prey. This system is a generalization of the two-species Lotka-Volterra predator-prey model. Firstly, we consider the asymptotical stability of equilibrium points to the system of ordinary differential equations type. Then, the global existence of solutions and the stability of equilibrium points to the system of weakly coupled reaction-diffusion type are discussed. Finally, the existence of nonnegative classical global solutions to the system of strongly coupled reaction-diffusion type is investigated when the space dimension is less than 6, and the global asymptotic stability of unique positive equilibrium point of the system is proved by constructing Lyapunov functions.

\section{Introduction and Mathematical Model}

The predator-prey model as, which follows, the ordinary differential equation system

$$
\begin{gathered}
\frac{d u}{d t}=\left(b_{1}+b_{2} u-b_{3} u^{2}\right) u-b_{4} u v, \\
\frac{d v}{d t}=-c v+(\alpha u-\beta v) v
\end{gathered}
$$

is said to be the general Lotka-Volterra predator-prey model in [1-3], and to be cubic predator-prey system in [4], where $u, v$ are the population densities of prey and predator species at time $t$, respectively. $b_{3}, b_{4}, c, \alpha, \beta$ are positive constants, $b_{1}$ is nonnegative as the intrinsic growth rate of prey population, and the sign of $b_{2}$ is undetermined. $c$ is the net 
mortality rate of predator population, and the survival of predator species is dependent on the survival state of prey species, and $b_{2} u-b_{3} u^{2}, \beta v$ are the respective density restriction terms of prey and predator species. $b_{4} u$ is the predation rate of the predator, and $\alpha u$ is the conversion rate of the predator. In [4], three questions about system (1.1) are discussed: the stability of nonnegative equilibrium points, and the existence, as well as numbers of limit cycle.

Referring to [5], we establish cubic predator-prey system with stage structure for the prey as follows:

$$
\begin{gathered}
\frac{\mathrm{d} x_{1}}{\mathrm{~d} t}=\eta_{1} x_{2}-r_{1} x_{1}-\eta_{2} x_{1}+b_{2} x_{1}^{2}-b_{3} x_{1}^{3}-b_{4} x_{1} x_{3}, \\
\frac{\mathrm{d} x_{2}}{\mathrm{~d} t}=\eta_{2} x_{1}-r_{2} x_{2} \\
\frac{\mathrm{d} x_{3}}{\mathrm{~d} t}=-c x_{3}+\left(\alpha x_{1}-\beta x_{3}\right) x_{3}
\end{gathered}
$$

where $x_{1}$ and $x_{2}$ are the population densities of the immature and mature prey species, respectively, and $x_{3}$ denotes the density of the predator species. The predators live only on the immature prey species, as well as the survival of the predator species is dependent on the survival state of the immature prey species. $\eta_{1}, \eta_{2}, r_{1}, r_{2}, b_{3}, b_{4}, c, \alpha, \beta$ are positive constants, and the sign of $b_{2}$ is undetermined. $\eta_{1}$ and $r_{1}$ are the birth rate and the mortality rate of the immature prey species, respectively. $r_{2}$ and $c$ are the net mortality rate of the mature prey population and the predator population, and $\eta_{2}$ is the conversion rate of the immature prey to the mature prey species. $b_{2} x_{1}-b_{3} x_{1}^{2}$ and $\beta x_{3}$ are the respective density restriction terms of the immature prey species and predator species. $b_{4} x_{1}$ is the predation rate of the predator to the immature prey population, and $\alpha x_{1}$ is the conversion rate of the predator.

Using the scaling

$$
u_{1}=\frac{\alpha}{r_{2}} x_{1}, \quad u_{2}=\frac{\alpha}{\eta_{2}} x_{2}, \quad u_{3}=\frac{\beta}{r_{2}} x_{3}, \quad d \tau=r_{2} d t,
$$

and redenoting $\tau$ by $t$, system (1.2) reduces to

$$
\begin{gathered}
\frac{\mathrm{d} u_{1}}{\mathrm{~d} t}=a_{0} u_{2}-a_{1} u_{1}+a_{2} u_{1}^{2}-a_{3} u_{1}^{3}-k u_{1} u_{3}, \\
\frac{\mathrm{d} u_{2}}{\mathrm{~d} t}=u_{1}-u_{2}, \\
\frac{\mathrm{d} u_{3}}{\mathrm{~d} t}=\left(-b+u_{1}-u_{3}\right) u_{3},
\end{gathered}
$$

where $a_{0}=\eta_{1} \eta_{2} / r_{2}^{2}, a_{1}=\left(r_{1}+\eta_{2}\right) / r_{2}, a_{2}=b_{2} / \alpha, a_{3}=b_{3} / r_{2}, k=b_{4} / \beta$, and $b=c / r_{2}$ are positive constants, and $a_{2}=b_{2} / \alpha$ is undetermined to the sign.

To take into account the inhomogeneous distribution of the predators and prey in different spatial locations within a fixed bounded domain $\Omega \subset \mathbb{R}^{N}$ at any given time, and the natural tendency of each species to diffuse to areas of smaller population concentration, 
we derive the following PDE system of reaction-diffusion type:

$$
\begin{gathered}
u_{1 t}-d_{1} \Delta u_{1}=a_{0} u_{2}-a_{1} u_{1}+a_{2} u_{1}^{2}-a_{3} u_{1}^{3}-k u_{1} u_{3}, \quad x \in \Omega, t>0, \\
u_{2 t}-d_{2} \Delta u_{2}=u_{1}-u_{2}, \quad x \in \Omega, t>0, \\
u_{3 t}-d_{3} \Delta u_{3}=\left(-b+u_{1}-u_{3}\right) u_{3}, \quad x \in \Omega, t>0, \\
\partial_{\eta} u_{i}=0, \quad i=1,2,3, \quad x \in \partial \Omega, t>0, \\
u_{i}(x, 0)=u_{i 0}(x) \geq 0, \quad i=1,2,3, x \in \Omega,
\end{gathered}
$$

where $\partial_{\eta}=\partial / \partial_{\eta}, \eta$ is the unit outward normal vector of the boundary $\partial \Omega$ which we will assume to be smooth. The homogeneous Neumann boundary condition indicates that the above system is self-contained with zero population flux across the boundary. The positive constants $d_{1}, d_{2}$, and $d_{3}$ are said to be the diffusion coefficients, and the initial values $u_{i 0}(x)$ $(i=1,2,3)$ are nonnegative smooth functions.

Note that, in recent years, there has been considerable interest to investigate the global behavior of a system of interacting populations by taking into account the effect of self as well as cross-diffusion. According to the ideas in [6-13], especially to [8, 9], the cross-diffusion term will be only included in the third equation, that is, the following cross-diffusion system:

$$
\begin{gathered}
u_{t}=\Delta\left(d_{1} u+\alpha_{11} u^{2}\right)+a_{0} v-a_{1} u+a_{2} u^{2}-a_{3} u^{3}-k u w, \quad x \in \Omega, t>0, \\
v_{t}=\Delta\left(d_{2} v+\alpha_{22} v^{2}\right)+u-v, \quad x \in \Omega, t>0, \\
w_{t}=\Delta\left(d_{3} w+\alpha_{31} u w+\alpha_{32} v w+\alpha_{33} w^{2}\right)+(-b+u-w) w, \quad x \in \Omega, t>0, \\
u_{\eta}(x, t)=v_{\eta}(x, t)=w_{\eta}(x, t)=0, \quad x \in \partial \Omega, t>0, \\
u(x, 0)=u_{0}(x) \geq 0, \quad v(x, 0)=v_{0}(x) \geq 0, \quad w(x, 0)=w_{0}(x) \geq 0, \quad x \in \Omega .
\end{gathered}
$$

In the above, $d_{i}, \alpha_{i i}(i=1,2,3), \alpha_{31}$, and $\alpha_{32}$ are positive constants. $d_{1}, d_{2}$ and $d_{3}$ are the diffusion rates of the three species, respectively. $\alpha_{i i}(i=1,2,3)$ are referred to as selfdiffusion pressures. $\alpha_{31}$ and $\alpha_{32}$ are cross-diffusion pressures. The term self-diffusion implies the movement of individuals from a higher to a lower concentration region. Cross-diffusion expresses the population fluxes of one species due to the presence of the other species. Generally, the value of the cross-diffusion coefficient may be positive, negative, or zero. The term positive cross-diffusion coefficient denotes the movement of the species in the direction of lower concentration of another species, and negative cross-diffusion coefficient denotes that one species tends to diffuse in the direction of higher concentration of another species [9].

The main purpose of this paper is to study the asymptotic behavior of the solutions of the reaction-diffusion system (1.5) and the global existence of the solution of the crossdiffusion system (1.6). But it is necessary to denonstrate that the conclusion for the existence of global solution of system (1.6) in this paper is the generalization of the work to LotkaVolterra competition model with cross-diffusion [11] and that the convergence of solution investigated in this paper which is not discussed in [11]. 
The paper will be organized as follows. In Section 2, we analyze the asymptotical stability of equilibrium points for the ODE system (1.4) via linearization and the Lyapunov method. In Section 3, we prove the global existence of solutions and the stability of the equilibrium points to the diffusion system (1.5). In Section 4, we investigate the existence of nonnegative classical global solutions by assuming $a_{0}, a_{1}, a_{2}, a_{3}, k, b$ to be positive constants only for the simplicity of calculation, and the global asymptotic stability of unique positive equilibrium point to the cross-diffusion system (1.6).

\section{Equilibrium Solution of the ODE System}

In this section we discuss the stability of unique positive equilibrium point for system (1.4). The following theorem shows that the solution of system (1.4) is bounded.

Theorem 2.1. Let $\left(u_{1}(t), u_{2}(t), u_{3}(t)\right)$ be the solution of system (1.4) with initial values $u_{i}(0)>$ $0(i=1,2,3)$, and let $[0, T)$ be the maximal existence interval of the solution. Then $0<u_{i}(t) \leq$ $M_{i}(i=1,2,3), t \in[0, T)$, where

$$
\begin{gathered}
M_{1}=\max \left\{u_{1}(0)+\left(a_{0}+a_{1}\right) u_{2}(0), C_{0}\left(1+\frac{a_{0}}{a_{1}}\right)\right\}, \\
M_{2}=\max \left\{\frac{u_{1}(0)}{a_{0}+a_{1}}, \frac{C_{0}}{a_{1}}\right\}, \\
M_{3}=\max \left\{u_{3}(0), M_{1}-b\right\} .
\end{gathered}
$$

The above $C_{0}$ is a positive constant depending only on $a_{0}, a_{1}, a_{2}, a_{3}$, and further $T=+\infty$.

Proof. It is easy to see that (1.4) has a unique positive local solution $\left(u_{1}(t), u_{2}(t), u_{3}(t)\right)$. Let $T \in(0,+\infty]$ be the maximal existence time of the solution, and combin $u_{1}$ and $u_{2}$ linearly, that is, $u_{1}+\left(a_{0}+a_{1}\right) u_{2}$, it follows from (1.4) that

$$
\frac{d}{d t}\left[u_{1}+\left(a_{0}+a_{1}\right) u_{2}\right] \leq-a_{1} u_{2}+a_{0} u_{1}+a_{2} u_{1}^{2}-a_{3} u_{1}^{3} .
$$

Using Young inequality, we can check that there exists a positive constant $C_{0}$ depending only on $a_{0}, a_{1}, a_{2}$, and $a_{3}$ such that

$$
a_{0} u_{1}+a_{2} u_{1}^{2}-a_{3} u_{1}^{3} \leq C_{0}-\frac{a_{1}}{a_{0}+a_{1}} u_{1}
$$

It follows that

$$
\frac{d}{d t}\left[u_{1}+\left(a_{0}+a_{1}\right) u_{2}\right] \leq C_{0}-\frac{a_{1}}{a_{0}+a_{1}}\left[u_{1}+\left(a_{0}+a_{1}\right) u_{2}\right]
$$


which implies that there exist $M_{1}$ and $M_{2}$ referring to (2.1) such that $0<u_{1} \leq M_{1}, 0<u_{2} \leq$ $M_{2}$, and $t \in[0, T)$.

Finally, we note that $d u_{3} / d t=\left(-b+u_{1}-u_{3}\right) u_{3} \leq\left(-b+M_{1}-u_{3}\right) u_{3}$. Let $M_{3}=$ $\max \left\{u_{3}(0), M_{1}-b\right\}$, then

$$
\frac{d\left(u_{3}-M_{3}\right)}{d t}+\left(u_{3}-M_{3}\right) u_{3} \leq 0 \quad u_{3}(0)-M_{3} \leq 0
$$

From the comparison inequality for the ODE, we have $u_{3}-M_{3} \leq 0, t \in[0, T)$.

Thus the solutions for system (1.4) are bounded. Further, from the extension theorem of solutions, we have $T=+\infty$.

By the simple calculation, the sufficient conditions for system (1.4) having a unique positive equilibrium point as follows:

(i) $a_{2}-k=2 \sqrt{a_{3}\left(a_{1}-a_{0}-k b\right)}>2 a_{3} b$; (ii) $\max \left\{a_{1}-a_{0},\left(a_{2}-2 a_{3} b\right) b\right\} \leq b k \leq\left(a_{2}-\right.$ $\left.a_{3} b\right) b$, where the left equal sign holds if and only if $a_{1}-a_{0}<\left(a_{2}-2 a_{3} b\right) b$; (iii) $a_{1}-a_{0} \leq$ $b\left(a_{2}-a_{3} b\right) \leq k b$; (iv) $a_{1}-a_{0}<k b \leq\left(a_{2}-2 a_{3} b\right) b$; (v) $k b<a_{1}-a_{0} \leq\left(a_{2}-a_{3} b\right) b$ and $a_{2}-k \geq \max \left\{2 a_{3} b, 2 \sqrt{a_{3}\left(a_{1}-a_{0}-k b\right)}\right\}$, where the second equal sign holds if and only if $a_{3} b^{2}>a_{1}-a_{0}-k b$; (vi) $2 \sqrt{a_{3}\left(a_{1}-a_{0}-k b\right)}<a_{2}-k \leq 2 a_{3} b$ and $k b<a_{1}-a_{0}<\left(a_{2}-a_{3} b\right) b$.

If one of the above conditions holds, then system (1.4) has the unique positive equilibrium point $\left(\bar{u}_{1}, \bar{u}_{2}, \bar{u}_{3}\right)$, where

$$
\bar{u}_{1}=\bar{u}_{2}=\frac{\left(a_{2}-k\right)+\sqrt{\left(a_{2}-k\right)^{2}+4 a_{3}\left(k b+a_{0}-a_{1}\right)}}{2 a_{3}}, \quad \bar{u}_{3}=\bar{u}_{1}-b .
$$

Theorem 2.2. System (1.4) has the unique positive equilibrium point $\left(\bar{u}_{1}, \bar{u}_{2}, \bar{u}_{3}\right)$ when one of the above conditions (i), (ii), (iii), (iv), (v), and (vi) holds. If $a_{1}-a_{0}<\left(\left(a_{2}^{2}-2 k a_{2}\right) / 4 a_{3}\right)+k b$ holds, then $\left(\bar{u}_{1}, \bar{u}_{2}, \bar{u}_{3}\right)$ is locally asymptotically stable.

Theorem 2.2 is easy to be obtained by using linearization; therefore, we omit its proof. The objective of this section is to prove the following result.

Theorem 2.3. System (1.4) has the unique positive equilibrium point $\left(\bar{u}_{1}, \bar{u}_{2}, \bar{u}_{3}\right)$ when one of the above conditions (i), (ii), (iii), (iv), (v), and (vi) holds. If $a_{1}-a_{0}<\min \left\{\left(\left(a_{2}^{2}-2 k a_{2}\right) / 4 a_{3}\right)+k b, k b-\right.$ $\left.a_{2} k / a_{3}\right\}$ holds, then $\left(\bar{u}_{1}, \bar{u}_{2}, \bar{u}_{3}\right)$ is globally asymptotically stable.

Proof. We make use of the general Lyapunov function

$$
V(u(t))=\sum_{i=1}^{3} \alpha_{i}\left(u_{i}-\bar{u}_{i}-\bar{u}_{i} \ln \frac{u_{i}}{\bar{u}_{i}}\right)
$$

where $\alpha_{i}(i=1,2,3)$ are positive constants. It holds that $V(u(t)) \geq 0$ for any $t>0$. Calculating the derivative along each solution of system (1.4), we have 


$$
\begin{aligned}
\frac{d V}{d t}= & \sum_{i=1}^{3} \alpha_{i} \frac{u_{i}-\bar{u}_{i}}{u_{i}} \frac{d u_{i}}{d t} \\
= & \alpha_{1} \frac{u_{1}-\bar{u}_{1}}{u_{1}} \\
& \times\left\{\frac{a_{0}}{\bar{u}_{1}}\left[u_{1}\left(u_{2}-\bar{u}_{2}\right)-u_{2}\left(u_{1}-\bar{u}_{1}\right)\right]-u_{1}\left(u_{1}-\bar{u}_{1}\right)\left(-a_{2}+a_{3} u_{1}+a_{3} \bar{u}_{1}\right)-k u_{1}\left(u_{3}-\bar{u}_{3}\right)\right\} \\
& +\alpha_{2} \frac{u_{2}-\bar{u}_{2}}{u_{2}} \frac{1}{\bar{u}_{2}}\left[u_{2}\left(u_{1}-\bar{u}_{1}\right)-u_{1}\left(u_{2}-\bar{u}_{2}\right)\right]+\alpha_{3} u_{3}\left[\left(u_{1}-\bar{u}_{1}\right)-\left(u_{3}-\bar{u}_{3}\right)\right] \frac{u_{3}-\bar{u}_{3}}{u_{3}} \\
= & -\alpha_{1}\left(-a_{2}+a_{3} u_{1}+a_{3} \bar{u}_{1}\right)\left(u_{1}-\bar{u}_{1}\right)^{2}-\alpha_{3}\left(u_{3}-\bar{u}_{3}\right)^{2} \\
& +\left(\alpha_{3}-k \alpha_{1}\right)\left(u_{1}-\bar{u}_{1}\right)\left(u_{3}-\bar{u}_{3}\right)+a_{0} \alpha_{1}\left(u_{1}-\bar{u}_{1}\right) \\
& \times\left[-\frac{u_{2}}{u_{1} \bar{u}_{1}}\left(u_{1}-\bar{u}_{1}\right)+\frac{1}{\bar{u}_{1}}\left(u_{2}-\bar{u}_{2}\right)\right]+\alpha_{2}\left(u_{2}-\bar{u}_{2}\right) \frac{\left(u_{1}-\bar{u}_{1}\right) u_{2}-\left(u_{2}-\bar{u}_{2}\right) u_{1}}{u_{2} \bar{u}_{2}} .
\end{aligned}
$$

Let $\alpha_{2}=a_{0} \alpha_{1}$ and $\alpha_{3}=k \alpha_{1}$. Then

$$
\begin{aligned}
\frac{d V}{d t}= & -\alpha_{1}\left(-a_{2}+a_{3} u_{1}+a_{3} \bar{u}_{1}\right)\left(u_{1}-\bar{u}_{1}\right)^{2}-\alpha_{3}\left(u_{3}-\bar{u}_{3}\right)^{2} \\
& -a_{0} \alpha_{1} \frac{1}{\bar{u}_{1}}\left[\sqrt{\frac{u_{2}}{u_{1}}}\left(u_{1}-\bar{u}_{1}\right)-\sqrt{\frac{u_{1}}{u_{2}}}\left(u_{2}-\bar{u}_{2}\right)\right]^{2}
\end{aligned}
$$

We observe that

$$
a_{1}-a_{0}<k b-\frac{a_{2} k}{a_{3}}
$$

is a sufficient condition of $-a_{2}+a_{3} u_{1}+a_{3} \bar{u}_{1}>0$. So, when condition (2.10) holds, we have

$$
\frac{d V}{d t} \leq 0
$$

Set $D=\left\{u \in \operatorname{Int} \mathbb{R}_{+}^{3}: d V / d t=0\right\}=\left\{\left(\bar{u}_{1}, \bar{u}_{2}, \bar{u}_{3}\right)\right\}$. According to the Lyapunov-LaSalle invariance principle [14], $\left(\bar{u}_{1}, \bar{u}_{2}, \bar{u}_{3}\right)$ is global asymptotic stability if inequality (2.10) and all conditions of Theorem 2.2 are satisfied. Theorem 2.3 is, thus, proved.

\section{Stability of the PDE System without Cross-Diffusion}

In this section, we first prove the global existence and uniform boundedness of solutions, then discuss the stability of unique positive equilibrium solution for the weakly coupled reactiondiffusion system (1.5). 
Denote that $F(\mathbf{u})=\left(f_{1}, f_{2}, f_{3}\right)^{\mathrm{T}}$, where $\mathbf{u}=\left(u_{1}, u_{2}, u_{3}\right), f_{1}=a_{0} u_{2}-a_{1} u_{1}+a_{2} u_{1}^{2}-a_{3} u_{1}^{3}-$ $k u_{1} u_{3}, f_{2}=u_{1}-u_{2}$, and $f_{3}=\left(-b+u_{1}-u_{3}\right) u_{3}$. It is easy to see that $f_{1}, f_{2}, f_{3} \in C^{1}\left(\bar{R}_{+}^{3}\right)$ with $\bar{R}_{+}^{3}=\left\{\left(u_{1}, u_{2}, u_{3}\right) \mid u_{i} \geq 0, i=1,2,3\right\}$. The standard PDE theory [15] shows that (1.5) has the unique solution $\left(u_{1}, u_{2}, u_{3}\right) \in\left[C(\bar{\Omega} \times[0, T)) \cap C^{2,1}(\Omega \times(0, T))\right]^{3}$, where $T \leq+\infty$ is the maximal existence time. The following theorem shows that the solution of (1.5) is uniformly bounded, and thus $T=+\infty$.

Theorem 3.1. Let $\left(u_{1}, u_{2}, u_{3}\right) \in\left[C(\bar{\Omega} \times[0, T)) \cap C^{2,1}(\Omega \times(0, T))\right]^{3}$ be the solution of system (1.5) with initial values $u_{i 0}(x) \geq 0(i=1,2,3)$, and let $T$ be the maximal existence time. Then $0 \leq u_{1}(x, t), u_{2}(x, t) \leq M_{1}, 0 \leq u_{3}(x, t) \leq M_{2}$, and $t \in[0, T)$, where $M_{1}$ is a positive constant depending only on $\Omega$ and all coefficients of $(1.5)$ and $\left\|u_{i 0}\right\|_{L^{\infty}(\Omega)}(i=1,2)$, $M_{2}=\max \left\{\left\|u_{30}\right\|_{L^{\infty}(\Omega)}, M_{1}-b\right\}$. Furthermore, $T=+\infty$ and $u_{i}(x, t)>0$ on $\bar{\Omega}$ for any $t>0$ if $u_{i 0} \geq(\not \equiv) 0(i=1,2,3)$.

Proof. Let $\left(u_{1}, u_{2}, u_{3}\right)$ be the solution of (1.5) with initial values $u_{i 0}(x) \geq 0(i=1,2,3)$. From the maximum principle for parabolic equations [16], it is not hard to verify that $u_{i}(x, t) \geq 0$ for $(x, t) \in \Omega \times[0, T)(i=1,2,3)$, where $T$ is the maximal existence time of the solution $\left(u_{1}, u_{2}, u_{3}\right)$. Furthermore, we know by the strong maximum principle that $u_{i}(x, t)>0$ on $\bar{\Omega}$ for all $t>0$ if $u_{i 0} \geq(\not \equiv) 0(i=1,2,3)$. Next we prove that the solution $\left(u_{1}, u_{2}, u_{3}\right)$ is bounded on $\Omega \times[0, T)$.

Integrating the first two equations of (1.5) over $\Omega$ and adding the results linearly, we have that, by Young inequality,

$$
\begin{aligned}
\frac{d}{d t} \int_{\Omega}\left[u_{1}+\left(a_{0}+a_{1}\right) u_{2}\right] d x & \leq \int_{\Omega}\left(a_{0} u_{1}-a_{1} u_{2}\right) d x+\int_{\Omega}\left(a_{2} u_{1}^{2}-a_{3} u_{1}^{3}\right) d x \\
& \leq-a_{1} \int_{\Omega} u_{2} d x+\int_{\Omega}\left[\left(a_{0}+\frac{a_{2}^{2}}{a_{3}}\right) u_{1}-a_{2} u_{1}^{2}\right] d x \\
& \leq C-\frac{a_{1}}{a_{0}+a_{1}} \int_{\Omega}\left[u_{1}+\left(a_{0}+a_{1}\right) u_{2}\right] d x
\end{aligned}
$$

for some positive constant $C$ depending only on the coefficients of (1.5). Therefore, $\left\|u_{1,2}(t)\right\|_{L^{1}(\Omega)}$ is bounded in [0, $\left.\infty\right)$. Using [17, Exercise 5 of Section 3.5], we obtain that $\left\|u_{1,2}(t)\right\|_{L^{\infty}(\Omega)}$ is also bounded in $[0, \infty)$. Now note that $\sup _{\bar{\Omega} \times[0, \infty)} u_{1,2}(x, t) \leq M_{1}$. The maximum principle gives $u_{3}(x, t) \leq \max \left\{\left\|u_{30}\right\|_{L^{\infty}(\Omega)}, M_{1}-b\right\}:=M_{2}$. The proof of Theorem 3.1 is completed.

In order to prove the global stability of unique positive equilibrium solution for system (1.5), we first recall the following lemma which can be found in $[7,17]$.

Lemma 3.2. Let $a$ and $b$ be positive constants. Assume that $\phi, \varphi \in C^{1}([a, \infty)), \varphi(t)>0$, and $\phi$ is bounded from below. If $\phi^{\prime}(t) \leq-b \varphi(t)$, and $\varphi^{\prime}(t) \leq K$ in $[a, \infty)$ for some constant $K$, then $\lim _{t \rightarrow \infty} \varphi(t)=0$. 
Let $0=\mu_{1}<\mu_{2}<\mu_{3}<\cdots$ be the eigenvalues of the operator $-\Delta$ on $\Omega$ with the homogeneous Neumann boundary condition, and let $E\left(\mu_{i}\right)$ be the eigenspace corresponding to $\mu_{i}$ in $C^{1}(\bar{\Omega})$. Denote that $\mathbf{X}=\left\{\mathbf{u} \in\left[C^{1}(\bar{\Omega})\right]^{3} \partial_{\eta} \mathbf{u}=0, x \in \partial \Omega\right\},\left\{\phi_{i j} ; j=1,2, \ldots, \operatorname{dim} E\left(\mu_{i}\right)\right\}$ is an orthonormal basis of $E\left(\mu_{i}\right)$, and $\mathbf{X}_{i j}=\left\{\mathbf{c} \cdot \phi_{i j} \mid \mathbf{c} \in R^{3}\right\}$. Then

$$
\mathbf{X}=\bigoplus_{i=1}^{\infty} \mathbf{X}_{i}, \quad \mathbf{X}_{i}=\bigoplus_{j=1}^{\operatorname{dim} E\left(\mu_{i}\right)} \mathbf{X}_{i j}
$$

Next we present the clear proof of the the global stability by two steps:

Step 1 (Local Stability). Let $\mathfrak{D}=\operatorname{diag}\left(d_{1}, d_{2}, d_{3}\right)$ and $\mathfrak{L}=\mathfrak{D}+F_{\mathbf{u}}(\overline{\mathbf{u}}) \mathfrak{D} \Delta+\left\{a_{i j}\right\}$, where

$$
F_{\mathbf{u}}(\overline{\mathbf{u}})=\left(\begin{array}{ccc}
-2 a_{3} \bar{u}_{1}^{2}+a_{2} \bar{u}_{1}-a_{0} & a_{0} & -k \bar{u}_{1} \\
1 & -1 & 0 \\
\bar{u}_{3} & 0 & -\bar{u}_{3}
\end{array}\right) \triangleq\left(\begin{array}{ccc}
a_{11} & a_{12} & a_{13} \\
a_{21} & a_{22} & a_{23} \\
a_{31} & a_{32} & a_{33}
\end{array}\right)
$$

The linearization of (1.5) at $\overline{\mathbf{u}}$ is

$$
\mathbf{u}_{t}=\mathfrak{L} \mathbf{u} .
$$

For each $i \geq 1, \mathbf{X}_{i}$ is invariant under the operator $\mathfrak{L}$, and $\lambda$ is an eigenvalue of $\mathfrak{L}$ on $\mathbf{X}_{i}$ if and only if it is an eigenvalue of the matrix $-\mu_{i} \mathfrak{D}+F_{\mathbf{u}}(\overline{\mathbf{u}})$.

The characteristic polynomial of $-\mu_{i} \mathfrak{D}+F_{\mathbf{u}}(\overline{\mathbf{u}})$ is given by

$$
\varphi_{i}(\lambda)=\lambda^{3}+A_{i} \lambda^{2}+B_{i} \lambda+C_{i}
$$

where

$$
\begin{aligned}
A_{i}= & \mu_{i}\left(d_{1}+d_{2}+d_{3}\right)-a_{11}-a_{33}+1, \\
B_{i}= & \mu_{i}^{2}\left(d_{1} d_{2}+d_{1} d_{3}+d_{2} d_{3}\right)+\mu_{i}\left[d_{1}\left(1-a_{33}\right)-d_{2}\left(a_{11}+a_{33}\right)+d_{3}\left(1-a_{11}\right)\right] \\
& +a_{11} a_{33}-a_{13} a_{31}-a_{33}-\left(a_{11}+a_{0}\right), \\
C_{i}= & \mu_{i}^{3} d_{1} d_{2} d_{3}+\mu_{i}^{2}\left(d_{1} d_{3}-a_{11} d_{2} d_{3}-a_{33} d_{1} d_{2}\right) \\
& -\mu_{i}\left[d_{1} a_{33}-d_{2}\left(a_{11} a_{33}-a_{13} a_{31}\right)+d_{3}\left(a_{11}+a_{0}\right)\right]+a_{33}\left(a_{11}+a_{0}\right)-a_{13} a_{31} .
\end{aligned}
$$

Thus

$$
H_{i}=A_{i} B_{i}-C_{i}=P_{3} \mu_{i}^{3}+P_{2} \mu_{i}^{2}+P_{1} \mu_{i}+P_{0}
$$


where $P_{0}, P_{1}, P_{2}$, and $P_{3}$ are given by

$$
\begin{aligned}
P_{0}= & \left(a_{11}+a_{33}\right)\left[a_{13} a_{31}+a_{33}-a_{11} a_{33}+\left(a_{11}+a_{0}\right)\right]-a_{33}\left(1+a_{0}\right)-\left(a_{11}+a_{0}\right), \\
P_{1}= & d_{1}\left[a_{11} a_{33}-a_{13} a_{31}-\left(a_{11}+a_{0}\right)\right]-d_{2}\left[\left(a_{11}+a_{0}\right)+a_{33}\right]+d_{3}\left(a_{11} a_{33}-a_{33}-a_{13} a_{31}\right) \\
& -\left(a_{11}+a_{33}-1\right)\left[d_{1}\left(1-a_{33}\right)-d_{2}\left(a_{11}+a_{33}\right)+d_{3}\left(1-a_{11}\right)\right], \\
P_{2}= & \left(d_{1}+d_{2}+d_{3}\right)\left[d_{1}\left(1-a_{33}\right)-d_{2}\left(a_{11}+a_{33}\right)+d_{3}\left(1-a_{11}\right)\right] \\
& -a_{11} d_{1}\left(d_{2}+d_{3}\right)+d_{2}\left(d_{1}+d_{3}\right)-a_{33} d_{3}\left(d_{1}+d_{2}\right), \\
P_{3}= & \left(d_{1}+d_{2}\right)\left(d_{1} d_{2}+d_{1} d_{3}+d_{2} d_{3}\right)+d_{3}^{2}\left(d_{1}+d_{2}\right) .
\end{aligned}
$$

According to the Routh-Hurwitz criterion [18], for each $i \geq 1$, the three roots $\lambda_{i, 1}, \lambda_{i, 2}, \lambda_{i, 3}$ of $\varphi_{i}(\lambda)=0$ all have negative real parts if and only if $A_{i}>0, C_{i}>0$, and $H_{i}>0$. Noting that $a_{33}<0$ and $a_{13} a_{31}<0$, the three roots have negative real parts if $a_{11}+a_{0}<0$. A direct calculation shows that $a_{11}+a_{0}$ is negative if

$$
a_{1}-a_{0}<\frac{a_{2}^{2}-2 a_{2} k}{4 a_{3}}+k b
$$

Now we can conclude that there exists a positive constant $\delta$ such that

$$
\operatorname{Re}\left\{\lambda_{i, 1}\right\}, \operatorname{Re}\left\{\lambda_{i, 2}\right\}, \operatorname{Re}\left\{\lambda_{i, 3}\right\} \leq-\delta, \quad i \geq 1 .
$$

In fact, let $\lambda=\mu_{i} \xi$, then

$$
\varphi_{i}(\lambda)=\mu_{i}^{3} \xi^{3}+A_{i} \mu_{i}^{2} \xi^{2}+B_{i} \mu_{i} \xi+C_{i} \triangleq \tilde{\varphi}_{i}(\xi)
$$

Since $\mu_{i} \rightarrow \infty$ as $i \rightarrow \infty$, it follows that

$$
\lim _{i \rightarrow \infty} \frac{\tilde{\varphi}_{i}(\xi)}{\mu_{i}^{3}}=\xi^{3}+\left(d_{1}+d_{2}+d_{3}\right) \xi^{2}+\left(d_{1} d_{2}+d_{2} d_{3}+d_{1} d_{3}\right) \xi+d_{1} d_{2} d_{3} \triangleq \tilde{\varphi}(\xi) .
$$

It is easy to see that $d_{1}, d_{2}, d_{3}$ are the three roots of $\tilde{\varphi}(\xi)=0$. Thus, there exists a positive constant $\bar{\delta}$ such that

$$
\operatorname{Re}\left\{-d_{1}\right\}, \operatorname{Re}\left\{-d_{2}\right\}, \operatorname{Re}\left\{-d_{3}\right\} \leq-\bar{\delta}
$$

By continuity, we see that there exists $i_{0}$ such that the three roots $\xi_{i, 1}, \xi_{i, 2}, \xi_{i, 3}$ of $\tilde{\varphi}_{i}(\xi)=0$ satisfy

$$
\operatorname{Re}\left\{\xi_{i, 1}\right\}, \operatorname{Re}\left\{\xi_{i, 2}\right\}, \operatorname{Re}\left\{\xi_{i, 3}\right\} \leq-\frac{\bar{\delta}}{2}, \quad i \geq i_{0}
$$


So

$$
\operatorname{Re}\left\{\lambda_{i, 1}\right\}, \operatorname{Re}\left\{\lambda_{i, 2}\right\}, \operatorname{Re}\left\{\lambda_{i, 3}\right\} \leq-\frac{\mu_{i} \bar{\delta}}{2} \leq-\frac{\widehat{\delta}}{2}, \quad i \geq i_{0}
$$

Let

$$
-\widetilde{\delta}=\max _{1 \leq i \leq i_{0}}\left\{\operatorname{Re}\left\{\lambda_{i, 1}\right\}, \operatorname{Re}\left\{\lambda_{i, 2}\right\}, \operatorname{Re}\left\{\lambda_{i, 3}\right\}\right\},
$$

then $\tilde{\delta}>0$, and (3.10) holds for $\delta=\min \{\tilde{\delta}, \widehat{\delta} / 2\}$.

Consequently, the spectrum of $\mathfrak{L}$, consisting only of eigenvalues, lies in $\{\operatorname{Re} \lambda \leq-\delta\}$ if (3.9) holds, and the local stability of $\overline{\mathbf{u}}$ follows [19, Theorem 5.1.1].

Step 2 (Global Stability). In the following, $C$ denotes a generic positive constant which does not depend on $x \in \bar{\Omega}$ and $t \geq 0$. Let $\mathbf{u}$ be the unique positive solution. Then it follows from Theorem 3.1 that $\mathbf{u}(\cdot, t)$ is bounded uniformly on $\bar{\Omega}$, that is, $\left\|u_{i}(\cdot, t)\right\|_{\infty} \leq C$ for all $t \geq 0$. By [20, Theorem $A_{2}$,

$$
\left\|u_{i}(\cdot, t)\right\|_{C^{2, \alpha}(\bar{\Omega})} \leq C, \quad \forall t \geq 1
$$

Define the Lyapunov function

$$
\begin{aligned}
E(t)= & \int_{\Omega}\left(u_{1}-\bar{u}_{1}-\bar{u}_{1} \ln \frac{u_{1}}{\bar{u}_{1}}\right) d x+a_{0} \int_{\Omega}\left(u_{2}-\bar{u}_{2}-u_{2} \ln \frac{u_{2}}{\bar{u}_{2}}\right) d x \\
& +k \int_{\Omega}\left(u_{3}-\bar{u}_{3}-\bar{u}_{3} \ln \frac{u_{3}}{\bar{u}_{3}}\right) d x .
\end{aligned}
$$

Then $E(t) \geq 0$ for all $t>0$. Using (1.5) and integrating by parts, we have

$$
\begin{aligned}
E^{\prime}(t)= & \int_{\Omega}\left(\frac{u_{1}-\bar{u}_{1}}{u_{1}} u_{1 t}+a_{0} \frac{u_{2}-\bar{u}_{2}}{u_{2}} u_{2 t}+k \frac{u_{3}-\bar{u}_{3}}{u_{3}} u_{3 t}\right) d x \\
= & -\int_{\Omega}\left(\frac{d_{1} \bar{u}_{1}}{u_{1}^{2}}\left|\nabla u_{1}\right|^{2}+a_{0} \frac{d_{2} \bar{u}_{2}}{u_{2}^{2}}\left|\nabla u_{2}\right|^{2}+k \frac{d_{3} \bar{u}_{3}}{u_{3}^{2}}\left|\nabla u_{3}\right|^{2}\right) d x \\
& +\int_{\Omega}\left[\frac{u_{1}-\bar{u}_{1}}{u_{1}} f_{1}(\mathbf{u})+a_{0} \frac{u_{2}-\bar{u}_{2}}{u_{2}} f_{2}(\mathbf{u})+k \frac{u_{3}-\bar{u}_{3}}{u_{3}} f_{3}(\mathbf{u})\right] d x \\
\leq & \int_{\Omega}\left\{-\left(-a_{2}+a_{3} u_{1}+a_{3} \bar{u}_{1}\right)\left(u_{1}-\bar{u}_{1}\right)^{2}-k\left(u_{3}-\bar{u}_{3}\right)^{2}\right. \\
& +a_{0}\left(u_{1}-\bar{u}_{1}\right)\left[-\frac{u_{2}}{u_{1} \bar{u}_{1}}\left(u_{1}-\bar{u}_{1}\right)+\frac{1}{\bar{u}_{1}}\left(u_{2}-\bar{u}_{2}\right)\right] \\
& \left.+a_{0}\left(u_{2}-\bar{u}_{2}\right) \frac{\left(u_{1}-\bar{u}_{1}\right) u_{2}-\left(u_{2}-\bar{u}_{2}\right) u_{1}}{u_{2} \bar{u}_{2}}\right\} d x
\end{aligned}
$$


Boundary Value Problems

$$
\begin{aligned}
= & \int_{\Omega}\left[-\left(-a_{2}+a_{3} u_{1}+a_{3} \bar{u}_{1}\right)\left(u_{1}-\bar{u}_{1}\right)^{2}-k\left(u_{3}-\bar{u}_{3}\right)^{2}\right] d x \\
& -\frac{a_{0}}{\bar{u}_{1}} \int_{\Omega}\left[\sqrt{\frac{u_{2}}{u_{1}}}\left(u_{1}-\bar{u}_{1}\right)-\sqrt{\frac{u_{1}}{u_{2}}}\left(u_{2}-\bar{u}_{2}\right)\right]^{2} d x \\
\leq & -\int_{\Omega}\left(-a_{2}+a_{3} u_{1}+a_{3} \bar{u}_{1}\right)\left(u_{1}-\bar{u}_{1}\right)^{2} d x-k \int_{\Omega}\left(u_{3}-\bar{u}_{3}\right)^{2} d x .
\end{aligned}
$$

Taking $l_{1}=-a_{2}+a_{3} \bar{u}_{1}$ and $l_{3}=k$, we have that

$$
E^{\prime}(t) \leq-l_{1} \int_{\Omega}\left(u_{1}-\bar{u}_{1}\right)^{2} d x-l_{3} \int_{\Omega}\left(u_{3}-\bar{u}_{3}\right)^{2} d x
$$

where $l_{1}>0$ holds for

$$
\left(a_{1}-a_{0}\right)<\frac{\left(a_{3} b-a_{2}\right) k}{a_{3}} .
$$

From Theorem 3.1 the solution $u_{i}$ of (1.5) is bounded, and so are the derivatives of $\left(u_{1}-\bar{u}_{1}\right)^{2}$ and $\left(u_{3}-\bar{u}_{3}\right)^{2}$ by equations in (1.5). Applying Lemma 3.2, we obtain

$$
\lim _{t \rightarrow \infty} \int_{\Omega}\left(u_{1}-\bar{u}_{1}\right)^{2} d x=0, \quad \lim _{t \rightarrow \infty} \int_{\Omega}\left(u_{3}-\bar{u}_{3}\right)^{2} d x=0
$$

As $u_{i}^{2} \leq C$, it follows that

$$
\begin{aligned}
E^{\prime}(t) & \leq-\int_{\Omega}\left(\frac{d_{1} \bar{u}_{1}}{u_{1}^{2}}\left|\nabla u_{1}\right|^{2}+a_{0} \frac{d_{2} \bar{u}_{2}}{u_{2}^{2}}\left|\nabla u_{2}\right|^{2}+k \frac{d_{3} \bar{u}_{3}}{u_{3}^{2}}\left|\nabla u_{3}\right|^{2}\right) d x \\
& \leq-C \int_{\Omega}\left(\left|\nabla u_{1}\right|^{2}+\left|\nabla u_{2}\right|^{2}+\left|\nabla u_{3}\right|^{2}\right) d x \\
& \triangleq-\varphi(t) .
\end{aligned}
$$

Using inequality (3.17) and system (1.5), the derivative of $\varphi(t)$ is bounded in $[1, \infty)$. From Lemma 3.2, we conclude that $\varphi(t) \rightarrow 0$ as $t \rightarrow \infty$. Therefore

$$
\lim _{t \rightarrow \infty} \int_{\Omega}\left(\left|\nabla u_{1}\right|^{2}+\left|\nabla u_{2}\right|^{2}+\left|\nabla u_{3}\right|^{2}\right) d x=0
$$

Using the Poincaré inequality yields

$$
\lim _{t \rightarrow \infty} \int_{\Omega}\left(u_{1}-\tilde{u}_{1}\right)^{2} d x=\lim _{t \rightarrow \infty} \int_{\Omega}\left(u_{2}-\tilde{u}_{2}\right)^{2} d x=\lim _{t \rightarrow \infty} \int_{\Omega}\left(u_{3}-\tilde{u}_{3}\right)^{2} d x=0,
$$


where $\tilde{u}_{i}(t)=(1 /|\Omega|) \int_{\Omega} u_{i} d x, i=1,2,3$. Thus, it follows from (3.22) and (3.25) that

$$
|\Omega|\left(\tilde{u}_{1}(t)-\bar{u}_{1}\right)^{2}=\int_{\Omega}\left(\bar{u}_{1}-\bar{u}_{1}\right)^{2} d x \leq 2 \int_{\Omega}\left(\tilde{u}_{1}-u_{1}\right)^{2} d x+2 \int_{\Omega}\left(u_{1}-\bar{u}_{1}\right)^{2} d x \longrightarrow 0
$$

as $t \rightarrow \infty$. So we have $\tilde{u}_{1}(t) \rightarrow \bar{u}_{1}$ as $t \rightarrow \infty$. Similarly, $\tilde{u}_{3}(t) \rightarrow \bar{u}_{3}$ as $t \rightarrow \infty$. Therefore, there exists a sequence $\left\{t_{m}\right\}$ with $t_{m} \rightarrow \infty$ such that $\tilde{u}_{1}^{\prime}\left(t_{m}\right) \rightarrow 0$. As $\tilde{u}_{2}\left(t_{m}\right)$ is bounded, there exists a subsequence of $\left\{t_{m}\right\}$, still denoted by the same notation, and nonnegative constant $\widehat{u}_{2}$ such that

$$
\tilde{u}_{2}\left(t_{m}\right) \longrightarrow \widehat{u}_{2}
$$

At $t=t_{m}$, from the first equation of (1.5), we have

$$
\begin{aligned}
|\Omega| \tilde{u}_{1}^{\prime}\left(t_{m}\right)= & \left.\int_{\Omega} u_{1 t} d x\right|_{t_{m}}=\left.\int_{\Omega}\left(d \Delta u_{1}+f_{1}(u)\right) d x\right|_{t_{m}} \\
= & \left.\int_{\Omega} \frac{a_{0}}{\overline{\bar{u}}_{1}}\left[u_{1}\left(u_{2}-\bar{u}_{2}\right)-u_{2}\left(u_{1}-\bar{u}_{1}\right)\right] d x\right|_{t_{m}} \\
& +\int_{\Omega}-\left.u_{1}\left(u_{1}-\bar{u}_{1}\right)\left(-a_{2}+a_{3} u_{1}+\bar{u}_{1}\right) d x\right|_{t_{m}} \\
& +\int_{\Omega}-\left.k u_{1}\left(u_{3}-\bar{u}_{3}\right) d x\right|_{t_{m}} \longrightarrow 0 .
\end{aligned}
$$

In view of (3.22) and (3.27), it follows from (3.28) that $\widehat{u}_{2}=\bar{u}_{2}$, thus

$$
\lim _{m \rightarrow \infty} \tilde{u}_{2}\left(t_{m}\right)=\bar{u}_{2}
$$

According to (3.17), there exists a subsequence of $\left\{t_{m}\right\}$, denoted still by $\left\{t_{m}\right\}$, and nonnegative functions $w_{i} \in C^{2}(\bar{\Omega})$, such that

$$
\lim _{m \rightarrow \infty}\left\|u_{i}\left(\cdot, t_{m}\right)-w_{i}(\cdot)\right\|_{C^{2}(\bar{\Omega})}=0, \quad i=1,2,3
$$

In view of (3.29) and noting that in fact $\tilde{u}_{1}(t) \rightarrow \bar{u}_{1}$ and $\tilde{u}_{3}(t) \rightarrow \bar{u}_{3}$, we know that $w_{i} \equiv \bar{u}_{i}, i=$ $1,2,3$. Therefore,

$$
\lim _{m \rightarrow \infty}\left\|u_{i}\left(\cdot, t_{m}\right)-\bar{u}_{i}\right\|_{C^{2}(\bar{\Omega})}=0, \quad i=1,2,3
$$

The global asymptotic stability of $\overline{\mathbf{u}}$ follows from (3.31) and the local stability of $\overline{\mathbf{u}}$.

Theorem 3.3. System (1.5) has the unique positive equilibrium point $\left(\bar{u}_{1}, \bar{u}_{2}, \bar{u}_{3}\right)$ when one of the conditions (i), (ii), (iii), (iv), (v), and (vi) in Section 2 holds. If (3.9) and (3.21) hold, then $\left(\bar{u}_{1}, \bar{u}_{2}, \bar{u}_{3}\right)$ is globally asymptotically stable. 


\section{Global Existence of Classical Solutions and Convergence}

In this section, we discuss the existence of nonnegative classical global solutions and the global asymptotic stability of unique positive equilibrium point of system (1.6).

Some notations throughout this section are as follows: $Q_{T}=\Omega \times[0, T), u \in$ $W_{p}^{k}(\Omega)$ means that $D^{\alpha} u \in L^{p}(\Omega)$ for any $|\alpha| \leq k$ with $\alpha=\left(\alpha_{1}, \alpha_{2}, \ldots, \alpha_{n}\right)$, $\|u\|_{W_{p}^{k}(\Omega)}=$ $\left(\int_{\Omega} \sum_{|\alpha| \leq k}\left|D^{\alpha} u\right|^{p} d x\right)^{1 / p}, u \in W_{p}^{2,1}\left(Q_{T}\right)$ means that $u, u_{x_{i}}, u_{x_{i} x_{j}}(i, j=1,2, \ldots, n)$ and $u_{t}$ are in $L^{p}\left(Q_{T}\right),\|u\|_{W_{p}^{2,1}\left(Q_{T}\right)}=\left(\int_{Q_{T}}\left(|u|^{p}+|D u|^{p}+\left|D^{2} u\right|^{p}+\left|u_{t}\right|^{p}\right) d x d t\right)^{1 / p}$, and $\|u\|_{V_{2}\left(Q_{T}\right)}=$ $\sup _{0 \leq t \leq T}\|u(\cdot, t)\|_{L^{2}(\Omega)}+\|\nabla u\|_{L^{2}\left(Q_{T}\right)}$ with $V_{2}\left(Q_{T}\right)=L^{\infty}\left((0, T), L^{2}(\Omega)\right) \cap W_{2}^{1,0}\left(Q_{T}\right)$.

To obtain $C^{2+\alpha, 1+\alpha / 2}\left(\bar{Q}_{T}\right)$ normal estimates of the solution for (1.6), we present a series of lemmas in the following.

Lemma 4.1. Let $(u, v, w)$ be the solution of (1.6). Then there exists a positive constant $M_{0}(\geq 1)$ such that

$$
0 \leq u, v \leq M_{0}, \quad 0 \leq w, \quad \forall t \geq 0 .
$$

Proof. By applying the comparison principle [20] to system (1.6), we have $u \geq 0, v \geq 0$, and $w \geq 0$ in $\bar{Q}_{T}$. To prove that $u, v \leq M_{0}$ in the following, we consider the auxiliary problem

$$
\begin{gathered}
u_{t}-\left(d_{1}+2 \alpha_{11} u\right) \Delta u+2 \alpha_{11} \sum_{i=1}^{n} u_{x_{i}} u_{x_{i}}=f_{1}, \quad x \in \Omega, t>0, \\
v_{t}-\left(d_{2}+2 \alpha_{22} v\right) \Delta v+2 \alpha_{22} \sum_{i=1}^{n} v_{x_{i}} v_{x_{i}}=f_{2}, \quad x \in \Omega, t>0, \\
\partial_{\eta} u(x, t)=\partial_{\eta} v(x, t)=0, \quad x \in \partial \Omega, t>0, \\
u(x, 0)=u_{0}(x) \geq(\not \equiv) 0, \quad v(x, 0)=v_{0}(x) \geq(\not \equiv) 0, \quad x \in \Omega .
\end{gathered}
$$

Notice that the functions $f_{1}$ and $f_{2}$ are sufficiently smooth in $\mathbb{R}^{2}$, and are quasimonotone in $\mathbb{R}_{+}^{2}$. Let $(0,0)$ and $(M, N)$ be a pair of upper-lower solutions for $(4.2)$, where $M$ and $N$ are positive constants. Direct calculation with inequalities

$$
\begin{gathered}
a_{0} N-a_{1} M+a_{2} M^{2}-a_{3} M^{3} \leq 0, \\
M-N \leq 0, \\
u_{0} \leq M, \quad v_{0} \leq N
\end{gathered}
$$

yields $M=\max \left\{\left(a_{2}+\sqrt{a_{2}^{2}+4 a_{3}\left|a_{0}-a_{1}\right|}\right) / 2 a_{3},\left\|u_{0}\right\|_{L^{\infty}(\Omega)}\right\}$ and $N=\max \left\{M,\left\|v_{0}\right\|_{L^{\infty}(\Omega)}\right\}$. It follows that there exists $M_{0}=K \max \{M, N\} \geq 1$ for any $t \geq 0$, where $K$ is a big enough positive constant such that (4.1) holds. 
Lemma 4.2. Let $X=\left(d_{1}+\alpha_{11} u\right) u$, and $u \in L^{\infty}\left(Q_{T}\right)$ for the solution to following equation:

$$
\begin{gathered}
u_{t}=\Delta\left[\left(d_{1}+\alpha_{11} u\right) u\right]+f_{1}, \quad(x, t) \in \Omega \times(0, T), \\
\partial_{\eta} u=0, \quad(x, t) \in \partial \Omega \times(0, T), \\
u(x, 0)=u_{0}(x) \geq 0, \quad x \in \Omega,
\end{gathered}
$$

where $d_{1}, \alpha_{11}$ are positive constants and $0 \leq w \in L^{2}\left(Q_{T}\right)$. Then there exists a positive constant $C(T)$, depending on $\left\|u_{0}\right\|_{W_{2}^{1}(\Omega)}$ and $\left\|u_{0}\right\|_{L^{\infty}(\Omega)}$, such that

$$
\|X\|_{W_{2}^{2,1}\left(Q_{T}\right)} \leq C(T)
$$

Furthermore,

$$
\nabla X \in V_{2}\left(Q_{T}\right), \quad \nabla u \in L^{2(n+2) / n}\left(Q_{T}\right) .
$$

Proof. It is easy to check, from $X=\left(d_{1}+\alpha_{11} u\right) u$, that

$$
X_{t}=\left(d_{1}+2 \alpha_{11} u\right) \Delta X+C_{1}-C_{2} w ，
$$

where $C_{1}=\left(d_{1}+2 \alpha_{11} u\right)\left(a_{0} v-a_{1} u+a_{2} u^{2}-a_{3} u^{3}\right)$ and $C_{2}=k\left(d_{1}+2 \alpha_{11} u\right) u$. $C_{1}$ and $C_{2}$ are bounded in $Q_{T}$ from (4.1). Multiplying (4.7) by $-\Delta X$, and integrating by parts over $Q_{t}$, yields

$$
\begin{gathered}
\frac{1}{2} \int_{\Omega}|\nabla X(x, t)|^{2} d x-\frac{1}{2} \int_{\Omega}|\nabla X(x, 0)|^{2} d x+d_{1} \int_{Q_{t}}|\Delta X|^{2} d x d t \\
\quad \leq \int_{Q_{T}}\left|C_{1}+C_{2} w \| \Delta X\right| d x d t .
\end{gathered}
$$

Using Hölder inequality and Young inequality to estimate the right side of (4.8), we have

$$
\begin{aligned}
\left\|C_{1}+C_{2} w\right\|_{L^{2}\left(Q_{T}\right)}\|\Delta X\|_{L^{2}\left(Q_{T}\right)} & \leq m_{1}\left(1+\|w\|_{L^{2}\left(Q_{T}\right)}\right)\|\Delta X\|_{L^{2}\left(Q_{T}\right)} \\
& \leq \frac{m_{1}^{2}\left(1+M_{3}\right)^{2}}{2 d_{1}}+\frac{d_{1}}{2}\|\Delta X\|_{L^{2}\left(Q_{T}\right)}^{2}
\end{aligned}
$$

with some $m_{1}>0$. Substituting (4.9) into (4.8) yields

$$
\sup _{0 \leq t \leq T} \int_{\Omega}|\nabla X(x, t)|^{2} d x+d_{1} \int_{Q_{t}}|\Delta X|^{2} d x d t \leq m_{2},
$$


where $m_{2}$ depends on $\left\|u_{0}\right\|_{W_{2}^{1}(\Omega)}$ and $\left\|u_{0}\right\|_{L^{\infty}(\Omega)}$. Since $X \in L^{2}\left(Q_{T}\right)$, the elliptic regularity estimate [10, Lemma 2.3] yields

$$
\int_{Q_{T}}\left|X_{x_{i} x_{j}}\right|^{2} d x d t \leq m_{3}, \quad i, j=1, \ldots, n
$$

From (4.7), we have $X_{t} \in L^{2}\left(Q_{T}\right)$. Hence, $\|X\|_{W_{2}^{2,1}\left(Q_{T}\right)} \leq C(T)$. Moreover, the Sobolev embedding theorem shows that (4.6) holds.

Lemma 4.3 (Lemma 4.3 can be presented by combining Lemmas 2.3 and 2.4 in [11]). Let $p>$ $1, \tilde{p}=2+4 p / n(q+1)$, and let $w$ satisfy

$$
\sup _{0 \leq t \leq T}\|w\|_{L^{2 p /(p+1)}(\Omega)}+\|\nabla w\|_{L^{2}\left(Q_{T}\right)}<\infty
$$

and there exist positive constants $\beta \in(0,1)$ and $C_{T}$ such that $\int_{\Omega}|w(\cdot, t)|^{\beta} d x \leq C_{T}(\forall t \leq T)$. Then there exists a positive constant $M^{\prime}$ independent of $w$ but possibly depending on $n, \Omega, p, \beta$, and $C_{T}$ such that

$$
\|w\|_{L^{\tilde{q}}\left(Q_{T}\right)} \leq M^{\prime}\left\{1+\left(\sup _{0 \leq t \leq T}\|w(t)\|_{L^{2 p /(p+1)}(\Omega)}\right)^{4 p / n(p+1) \tilde{p}}\|\nabla w\|_{L^{2}\left(Q_{T}\right)}^{2 / \tilde{p}}\right\} .
$$

Finally, one proposes some standard embedding results which are important to obtain the $C^{2+\alpha, 1+\alpha / 2}\left(\bar{Q}_{T}\right)$ normal estimates of the solution for (1.6).

Lemma 4.4. Let $\Omega \subset \mathbb{R}^{N}$ be a fixed bounded domain and $\partial \Omega \subset C^{2}$. Then for all $u \in W_{q}^{2,1}\left(Q_{T}\right)$ with $q \geq 1$, one has

(1) $\|\nabla u\|_{L^{p}\left(Q_{T}\right)} \leq C\|u\|_{W_{q}^{2,1}\left(Q_{T}\right)}$, for all $1 \leq p \leq(n+2) q /(n+2-q), q<n+2$,

(2) $\|\nabla u\|_{L^{p}\left(Q_{T}\right)} \leq C\|u\|_{W_{q}^{2,1}\left(Q_{T}\right)}$, for all $1 \leq p \leq \infty, q=n+2$,

(3) $\|\nabla u\|_{C^{\alpha, \alpha / 2}\left(Q_{T}\right)} \leq C\|u\|_{W_{q}^{2,1}\left(Q_{T}\right)}$ for all $1-(n+2) / q \leq \alpha \leq 1, q>n+2$,

where $C$ is a positive constant dependent on $q, n, \Omega$, and $T$.

The main result about the global existence of nonnegative classical solution for the cross-diffusion system (1.6) is given as follows.

Theorem 4.5. Assume that $u_{0} \geq 0, v_{0} \geq 0$, and $w_{0} \geq 0$ satisfy homogeneous Neumann boundary conditions and belong to $C^{2+\lambda}(\bar{\Omega})$ for some $\lambda \in(0,1)$. Then system (1.6) has a unique nonnegative solution $u, v, w \in C^{2+\lambda, 1+\lambda / 2}(\bar{\Omega} \times[0, \infty))$ when the space dimension is $n \leq 5$. 
Proof.

Step 1. $L^{1}-, L^{2}$-Estimates and $L^{q}$-Estimates of $w$. Firstly, integrating the third equation of (1.6) over $\Omega$, we have

$$
\begin{aligned}
\frac{d}{d t} \int_{\Omega} w d x & \leq \frac{1}{2} \int_{\Omega} u^{2} d x-\frac{1}{2} \int_{\Omega} w^{2} d x \\
& \leq \frac{|\Omega|}{2} M_{0}^{2}-\frac{1}{2|\Omega|}\left(\int_{\Omega} w d x\right)^{2} .
\end{aligned}
$$

Thus

$$
\int_{\Omega} w d x \leq \max \left\{M_{0}|\Omega|, \int_{\Omega} w_{0} d x\right\}:=M_{1}, \quad \forall t \geq 0
$$

Furthermore

$$
\|w\|_{L^{1}\left(Q_{T}\right)} \leq \int_{0}^{T} M_{1} d t:=M_{2}
$$

Integrating (4.14) in $[0, T]$ and moving terms yield

$$
\|w\|_{L^{2}\left(Q_{T}\right)} \leq\left(M_{0}^{2}|\Omega| T+2\left\|w_{0}\right\|_{L_{1}(\Omega)}\right)^{1 / 2}:=M_{3}
$$

Secondly, multiplying the third equation of (1.6) by $q w^{q-1}(q>1)$ and integrating over $\Omega$, we have

$$
\begin{aligned}
\frac{d}{d t} \int_{\Omega} w^{q} d x \leq & -\frac{4(q-1) d_{3}}{q} \int_{\Omega}\left|\nabla\left(w^{q / 2}\right)\right|^{2} d x-\frac{8 q(q-1) \alpha_{33}}{(q+1)^{2}} \int_{\Omega}\left|\nabla\left(w^{(q+1) / 2}\right)\right|^{2} d x \\
& -(q-1) \alpha_{31} \int_{\Omega} \nabla u \cdot \nabla\left(w^{q}\right) d x-(q-1) \alpha_{32} \int_{\Omega} \nabla v \cdot \nabla\left(w^{q}\right) d x \\
& +q \int_{\Omega} w^{q}(-b+u-w) d x
\end{aligned}
$$


Integrating the above expression in $[0, t](t \leq T)$ yields

$$
\begin{aligned}
& \int_{\Omega} w^{q}(x, t) d x+\frac{4(q-1) d_{3}}{q} \int_{Q_{t}}\left|\nabla\left(w^{q / 2}\right)\right|^{2} d x d t+\frac{8 q(q-1) \alpha_{33}}{(q+1)^{2}} \int_{Q_{t}}\left|\nabla\left(w^{(q+1) / 2}\right)\right|^{2} d x d t \\
& \leq \int_{\Omega} w_{0}^{q}(x) d x-(q-1) \alpha_{31} \int_{Q_{t}} \nabla u \cdot \nabla\left(w^{q}\right) d x d t-(q-1) \alpha_{32} \int_{Q_{t}} \nabla v \cdot \nabla\left(w^{q}\right) d x d t \\
& \quad+q \int_{Q_{t}} w^{q}(-b+u-w) d x d t .
\end{aligned}
$$

Since $\nabla u \in L^{2(n+2) / n}\left(Q_{T}\right)$ from Lemma 4.2, and using Hölder inequality and Young inequality, we have

$$
\begin{aligned}
-(q- & 1) \alpha_{31} \int_{Q_{t}} \nabla u \cdot \nabla\left(w^{q}\right) d x d t-(q-1) \alpha_{32} \int_{Q_{t}} \nabla v \cdot \nabla\left(w^{q}\right) d x d t \\
\leq & \frac{2 q(q-1) \alpha_{31}}{q+1}\left|\int_{Q_{t}} w^{(q-1) / 2} \nabla\left(w^{(q+1) / 2}\right) \cdot \nabla u d x d t\right| \\
& +\frac{2 q(q-1) \alpha_{32}}{q+1}\left|\int_{Q_{t}} w^{(q-1) / 2} \nabla\left(w^{(q+1) / 2}\right) \cdot \nabla v d x d t\right| \\
\leq & \frac{2 q(q-1)}{q+1}\left(\alpha_{31}\|\nabla u\|_{L^{2(n+2) / n}\left(Q_{T}\right)}+\alpha_{32}\|\nabla v\|_{L^{2(n+2) / n}\left(Q_{T}\right)}\right) \\
& \times\left\|w^{(q-1) / 2}\right\|_{L^{n+2}\left(Q_{T}\right)}\left\|\nabla\left(w^{(q+1) / 2}\right)\right\|_{L^{2}\left(Q_{T}\right)} \\
\leq & C_{3}\left\|\nabla\left(w^{(q+1) / 2}\right)\right\|\left\|_{L^{2}\left(Q_{T}\right)}\right\| w^{(q-1) / 2} \|_{L^{n+2}\left(Q_{T}\right)} \\
\leq & C_{3} \varepsilon\left\|\nabla\left(w^{(q+1) / 2}\right)\right\|_{L^{2}\left(Q_{T}\right)}^{2}+\frac{C_{3}}{4 \varepsilon}\left\|w^{(q-1) / 2}\right\|_{L^{n+2}\left(Q_{T}\right)}^{2} .
\end{aligned}
$$

From (4.1) and $\alpha^{p} \beta \leq(p /(p+1)) \alpha^{p+1}+(1 /(p+1)) \beta^{p+1}(\alpha, \beta>0)$, it holds that

$$
\begin{aligned}
q \int_{Q_{t}} w^{q}(-b+u-w) d x d t & \leq q\left(-b+M_{0}\right) \int_{Q_{t}} w^{q} d x d t-q \int_{Q_{t}} w^{q+1} d x \\
& \leq q\left(-b+M_{0}\right)\left|Q_{T}\right|^{1 /(q+1)}\|w\|_{L^{q+1}\left(Q_{t}\right)}^{q}-q\|w\|_{L^{q+1}\left(Q_{t}\right)}^{q+1} \\
& \leq\left(-b+M_{0}\right)^{2}\left|Q_{T}\right| \triangleq C_{4} .
\end{aligned}
$$


Taking $\bar{w}=w^{(q+1) / 2}$ and selecting a proper $\varepsilon$ such that $C_{3} \varepsilon \leq 4 q(q-1) \alpha_{33} /(q+1)^{2}$, then applying (4.20) and (4.21) to (4.19) yields

$$
\begin{aligned}
& \int_{\Omega} \bar{w}^{2 q /(q+1)}(x, t) d x+\frac{4(q-1) d_{3}}{q} \int_{Q_{t}}\left|\nabla\left(\bar{w}^{q /(q+1)}\right)\right|^{2} d x d t+\frac{4 q(q-1) \alpha_{33}}{(q+1)^{2}} \int_{Q_{t}}|\nabla \bar{w}|^{2} d x d t \\
& \quad \leq\left\|w_{0}\right\|_{L^{q}(\Omega)}^{q}+\frac{C_{3}}{4 \varepsilon}\|\bar{w}\|_{L^{(q-1)(n+2) /(q+1)}\left(Q_{T}\right)}^{2(q-1) /(q+1)}+C_{4} \\
& \quad \leq C_{5}\left(1+\|\bar{w}\|_{L^{(q-1)(n+2) /(q+1)}\left(Q_{T}\right)}^{2(q-1) /(q+1)}\right) .
\end{aligned}
$$

Denote that $E \equiv \sup _{0<t<T} \int_{\Omega} \bar{w}^{2 q /(q+1)}(x, t) d x+\int_{Q_{T}}|\nabla \bar{w}|^{2} d x d t$. Then it follows from (4.22) that

$$
E \leq C_{6}\left(1+\|\bar{w}\|_{L^{(q-1)(n+2) /(q+1)}\left(Q_{T}\right)}^{2(q-1) /(q+1)}\right)
$$

It is easy to see that $2 q /(q+1)<2<\tilde{q}$ and $(q-1)(n+2) /(q+1)<\tilde{q}=2+4 q / n(q+1)$ for any $q<n(n+4) /\left(n^{2}-4\right)$; hence

$$
E \leq C_{7}\left(1+\|\bar{w}\|_{L^{\tilde{q}}\left(Q_{T}\right)}^{2(q-1) /(q+1)}\right) .
$$

Take $\beta=2 /(q+1) \in(0,1)$. Then it follows from $L^{1}(\Omega)$-estimates of $w$, namely $(4.15)$, that

$$
\|\bar{w}\|_{L^{\beta}(\Omega)}=\left(\int_{\Omega}|\bar{w}(\cdot, t)|^{\beta} d x\right)^{1 / \beta}=\|w\|_{L^{1}(\Omega)}^{1 / \beta} \leq M_{1}^{1 / \beta}, \quad \forall t \leq T .
$$

It follows from Lemma 4.3 and (4.24) that

$$
\begin{aligned}
E & \leq C_{7}\left[1+\left(M^{\prime}+M^{\prime} \sup _{0<t<T}\|\bar{w}(\cdot, t)\|_{L^{2 q /(q+1)}(\Omega)}^{4 q / n(q+) \tilde{q}}\|\nabla \bar{w}\|_{L^{2}\left(Q_{T}\right)}^{2 / \tilde{q}}\right)^{2(q-1) /(q+1)}\right] \\
& \leq C_{8}\left[1+\left(\sup _{0<t<T}\|\bar{w}(\cdot, t)\|_{L^{2 q /(q+1)}(\Omega)}^{2 q /(q+1)}\right)^{4(q-1) / n(q+1) \tilde{q}}\left(\|\nabla \bar{w}\|_{L^{2}\left(Q_{T}\right)}^{2}\right)^{2(q-1) /(q+1) \tilde{q}}\right] \\
& \leq C_{8}\left(1+E^{((2+n) / n \tilde{q}) \cdot 2((q-1) /(q+1-))}\right) .
\end{aligned}
$$

Since $((2+n) / n \tilde{q}) \cdot(2(q-1) /(q+1)) \in(0,1), E$ is bounded by contrary proof. It follows that $\|\bar{w}\|_{L^{\tilde{q}}\left(Q_{T}\right)}$ is bounded, that is, $w \in L^{(q+1) \tilde{q} / 2}\left(Q_{T}\right)$. It is easy to check that $(q+1) \tilde{q} / 2 \in$ $(1,2(n+1) /(n-2))$ for all $q<n(n+4) /\left(n^{2}-4\right)$ still denote $(q+1) \tilde{q} / 2$ by $q$, then

$$
w \in L^{q}\left(Q_{T}\right), \quad \forall q \in\left(1, \frac{2(n+1)}{n-2}\right) .
$$


Finally, we observe that $q=2$ satisfies $\left(n^{2}-4\right) q<n^{2}+4 n$ with $n=2,3,4,5$. So take $q=2$ for (4.17) and (4.19). Then there exists a positive constant $M_{4}$ such that

$$
\|w\|_{V_{2}\left(Q_{T}\right)} \leq M_{4}
$$

Step 2. $L^{\infty}$-Estimates of $w$. We rewrite the third equation of (1.6) as a linear parabolic equation

$$
\frac{\partial w}{\partial t}=\sum_{i, j=1}^{n} \frac{\partial}{\partial x_{i}}\left(a_{i j}(x, t) \frac{\partial w}{\partial x_{j}}\right)+\sum_{i=1}^{n} \frac{\partial}{\partial x_{i}}\left(a_{i}(x, t) w\right)-(b-u+w) w,
$$

where $a_{i j}(x, t)=\left(d_{3}+\alpha_{31} u+\alpha_{32} v+2 \alpha_{33} w\right) \delta_{i j}, a_{i}(x, t)=\alpha_{31}\left(\partial u / \partial x_{i}\right)+\alpha_{32}\left(\partial v / \partial x_{i}\right), \delta_{i j}$ are Kronecker symbols.

To apply the maximum principle [15, Theorem 7.1, page 181] to (4.15) to obtain $w \in$ $L^{\infty}\left(Q_{T}\right)$, we need to verify that the following conditions hold: (1) $\|w\|_{V_{2}\left(Q_{T}\right)}$ is bounded; (2) $\sum_{i, j=1}^{n} a_{i j}(x, t) \xi_{i} \xi_{j} \geq v \sum_{i=1}^{n} \xi_{i}^{2}$; (3) $\left\|\sum_{i=1}^{n} a_{i}^{2}(x, t), b-u+w\right\|_{L^{q, r}\left(Q_{T}\right)} \leq \mu_{1}$, where $v$ and $\mu_{1}$ are positive constants, and $q$ and $r$ satisfy

$$
\begin{gathered}
\frac{1}{r}+\frac{n}{2 q}=1-x, \quad 0<x<1 \\
q \in\left[\frac{n}{2(1-x)},+\infty\right) \quad r \in\left[\frac{1}{1-x},+\infty\right), \quad n \geq 2 .
\end{gathered}
$$

Next we verify conditions (1)-(3) in turn. From (4.28), condition (1) is true for $n \leq 5$. One can choose $v=d_{3}$ such that condition (2) holds. To verify condition (3), the first equation of (1.6) is written in the divergence form

$$
u_{t}=\nabla \cdot\left[\left(d_{1}+2 \alpha_{11} u\right) \nabla u\right]+a_{0} v-a_{1} u+a_{2} u^{2}-a_{3} u^{3}-k u w,
$$

where $d_{1}+2 \alpha_{11} u$ is bounded in $\bar{Q}_{T}$ by Lemma 4.1, and $a_{0} v-a_{1} u+a_{2} u^{2}-a_{3} u^{3}-k u w \in L^{q}\left(Q_{T}\right)$ for $q \in((n+2) / 2,2(n+1) /(n-2))$ from (4.27). Application of the Hölder continuity result [15, Theorem 10.1, page 204] to (4.19) yields

$$
u \in C^{\beta, \beta / 2}\left(\bar{Q}_{T}\right), \quad \beta \in(0,1) .
$$

Returning to (4.7), since $C_{1}+C_{2} w \in L^{q}\left(Q_{T}\right)$ for any $q \in((n+2) / 2,2(n+1) / n-2)$ by (4.1) and (4.27), and $d_{1}+\alpha_{11} u \in C^{\beta, \beta / 2}\left(\bar{Q}_{T}\right)$ by (4.32), then by applying the parabolic regularity theorem [15, Theorem 9.1, pages 341-342] to (4.7) we have

$$
X \in W_{q}^{2,1}\left(Q_{T}\right), \quad \forall q \in\left(\frac{n+2}{2}, \frac{2(n+1)}{n-2}\right) .
$$

Hence $\nabla X \in L^{(n+2) q /(n+2-q)}\left(Q_{T}\right)$ from Lemma 4.4, which shows that $\nabla u \in L^{(n+2) q /(n+2-q)}\left(Q_{T}\right)$. Similarly, $\nabla v \in L^{(n+2) q /(n+2-q)}\left(Q_{T}\right)$ by the second equation of (1.6). Now we can show that 
$|\nabla u|^{2},|\nabla v|^{2} \in L^{(n+2) q / 2(n+2-q)}\left(Q_{T}\right)$, which imply that $\sum_{i=1}^{n} a_{i}^{2}(x, t) \in L^{(n+2) q / 2(n+2-q)}\left(Q_{T}\right)$. In addition, $b-u+w$ obviously belongs to $L^{q}\left(Q_{T}\right)$. It follows that one can select $q=r=$ $(n+2) p / 2(n+2-p)$. Now the above three conditions are satisfied, and $w \in L^{\infty}\left(Q_{T}\right)$ from $[15$, Theorem 7.1, page 181]. Recalling Lemma 4.1, thus there exists a positive constant $M_{5}$ for any $T>0$ such that

$$
\|u\|_{L^{\infty}\left(Q_{T}\right)}, \quad\|v\|_{L^{\infty}\left(Q_{T}\right)}, \quad\|w\|_{L^{\infty}\left(Q_{T}\right)} \leq M_{5} .
$$

Step 3. The Proof of the Classical Solution $(u, v, w)$ of (1.6) in $Q_{T}$ for Any $T>0$. Because $\left(d_{1} u+\alpha_{11} u^{2} \alpha\right)_{t}=\left(d_{1}+2 \alpha_{11} u\right) \Delta\left(d_{1} u+\alpha_{11} u^{2}\right)+\left(d_{1}+2 \alpha_{11} u\right) f_{1}$, we have from (4.34) that $X=d_{1} u+\alpha_{11} u^{2} \in W_{q}^{2,1}\left(Q_{T}\right)$ for any $q>1$. So $\nabla X \in C^{\beta^{*}, \beta^{*} / 2}\left(\bar{Q}_{T}\right)$ for all $\beta^{*} \in(0,1)$. It follows from [15, Lemma 3.3, page 80] that $X \in C^{1+\beta^{*},\left(1+\beta^{*}\right) / 2}\left(\bar{Q}_{T}\right)$. And direct calculation $X=\left(d_{1}+\alpha_{11} u\right) u$ yields $u=\left(-d_{1}+\sqrt{d_{1}^{2}+4 \alpha_{11} X}\right) / 2 \alpha_{11}$. So we have

$$
u \in C^{1+\beta^{*},\left(1+\beta^{*}\right) / 2}\left(\bar{Q}_{T}\right), \quad \forall \beta^{*} \in(0,1) .
$$

The third equation of (1.6) can be written as

$$
w_{t}=\nabla \cdot\left[\left(d_{3}+\alpha_{31} u+\alpha_{32} v+2 \alpha_{33} w\right) \nabla w+\left(\alpha_{31} \nabla u+\alpha_{32} \nabla v\right) w\right]+(-b+u-w) w
$$

Summarizing the above conclusions that are proved, we know that $(-b+u-w) w \in L^{\infty}\left(Q_{T}\right)$ and $u, v, w, \nabla u, \nabla v$ are all bounded in $Q_{T}$. It follows from [15, Theorem 10.1, page 204] that there exists $\sigma_{1} \in(0,1)$ such that

$$
w \in C^{\sigma_{1}, \sigma_{1} / 2}\left(\bar{Q}_{T}\right)
$$

The proof of Lemma 4.2 is similar. Then we have $\nabla v \in L^{2}\left(Q_{T}\right)$, that is, $v \in V_{2}\left(Q_{T}\right)$. Applying the [13, Theorem 10.1, page 204] to the second equation (1.6), there exists $\sigma_{2} \in(0,1)$ such that

$$
v \in C^{\sigma_{2}, \sigma_{2} / 2}\left(\bar{Q}_{T}\right)
$$

Furthermore, applying Schauder estimate [15, page 320-321] yields $v \in C^{2+\sigma^{*}, 1+\sigma^{*} / 2}\left(\bar{Q}_{T}\right)$ for $\sigma^{*}=\min \left\{\sigma_{2}, \lambda\right\}$. Selecting $\alpha=\min \left\{\sigma_{1}, \sigma^{*}\right\}$ and using Sobolev embedding theorem, we have $C^{2+\sigma^{*}, 1+\sigma^{*} / 2}\left(\bar{Q}_{T}\right) \hookrightarrow C^{\alpha, \alpha / 2}\left(\bar{Q}_{T}\right)$. Still applying Schauder estimate, we have

$$
v \in C^{2+\sigma, 1+\sigma / 2}\left(\bar{Q}_{T}\right), \quad \sigma=\min \{\alpha, \lambda\}
$$

Let $\bar{w}=\left(d_{3}+\alpha_{31} u+\alpha_{32} v+\alpha_{33} w\right) w$. Then $\bar{w}$ satisfies

$$
\bar{w}=\left(d_{3}+\alpha_{31} u+\alpha_{32} v+2 \alpha_{33} w\right) \Delta \bar{w}+f(x, t),
$$


where $f(x, t)=\left(d_{3}+\alpha_{31} u+\alpha_{32} v+2 \alpha_{33} w\right)(-b+u-w) w+\left(\alpha_{31} u_{t}+\alpha_{32} v_{t}\right) w$. By (4.35)-(4.38), we have $d_{3}+\alpha_{31} u+\alpha_{32} v+2 \alpha_{33} w, f(x, t) \in C^{\alpha, \alpha / 2}\left(\bar{Q}_{T}\right)$. So applying Schauder estimate to (4.40) yields $\bar{w} \in C^{2+\sigma, 1+\sigma / 2}\left(\bar{Q}_{T}\right)$. Since $w=\left(-\left(d_{3}+\alpha_{31} u+\alpha_{32} v\right)+\sqrt{\left(d_{3}+\alpha_{31} u+\alpha_{32} v\right)^{2}+4 \alpha_{33} \bar{w}}\right) / 2 \alpha_{33}$, we have

$$
w \in C^{2+\sigma, 1+\sigma / 2}\left(\bar{Q}_{T}\right), \quad \sigma=\min \{\alpha, \lambda\}
$$

The first equation of (1.6) can be written as

$$
u_{t}=\left(d_{1}+2 \alpha_{11} u\right) \Delta u+g(x, t)
$$

where $g(x, t)=2 \alpha_{11}|\nabla u|^{2}+a_{0} v-a_{1} u+a_{2} u^{2}-a_{3} u^{3}-k u w$. By (4.35), (4.39), and (4.41), we have $d_{1}+2 \alpha_{11} u, g(x, t) \in C^{\alpha, \alpha / 2}\left(\bar{Q}_{T}\right)$. So applying Schauder estimate to (4.42) yields

$$
u \in C^{2+\sigma, 1+\sigma / 2}\left(\bar{Q}_{T}\right), \quad \sigma=\min \{\alpha, \lambda\}
$$

In particular, if $\lambda<\alpha$, then $\sigma=\lambda$; in other words, Theorem 4.5 is proved. For the case $\alpha<\lambda$, from Sobolev embedding theorem, we have $C^{2+\sigma, 1+\sigma / 2}\left(\bar{Q}_{T}\right) \hookrightarrow C^{\lambda, \lambda / 2}\left(\bar{Q}_{T}\right)$. Repeating the above bootstrap and Shauder estimate arguments, this completes the proof of Theorem 4.5. About space dimension $n=1$, see [21].

Theorem 4.6. System (1.6) has the unique positive equilibrium point $(\bar{u}, \bar{v}, \bar{w})$ when one of the conditions (i), (ii), (iii), (iv), (v), and (vi) in Section 2 holds. Let the space dimension be $n \leq 5$, and let the initial values $u_{0}, v_{0}, w_{0}$ be nonnegative smooth functions and satisfy the homogenous Neumann boundary conditions. If the following condition (4.44) holds, then the solution $(u, v, w)$ of (1.6) converges to $(\bar{u}, \bar{v}, \bar{w})$ in $L^{2}(\Omega)$ :

$$
4 \alpha \bar{u} d_{1} d_{2} d_{3}>\beta \bar{w}\left[\alpha \bar{u} \alpha_{32}^{2}\left(d_{1}+2 \alpha_{11} M_{0}\right)+\left(\alpha_{31} M_{0}\right)^{2}\left(d_{2}+2 \alpha_{22} M_{0}\right)\right]
$$

where $\alpha=2 / a_{0}^{2}+1 / 2 a_{3} \bar{u}$, and $\beta=\left(k+a_{3} \bar{u}\right) \alpha$.

Proof. Define the Lyapunov function

$$
H(t)=\alpha \int_{\Omega}\left(u-\bar{u}-\bar{u} \ln \frac{u}{\bar{u}}\right) d x+\frac{1}{2} \int_{\Omega}(v-\bar{v})^{2} d x+\beta \int_{\Omega}\left(w-\bar{w}-\bar{w} \ln \frac{w}{\bar{w}}\right) d x
$$

where $\alpha$ and $\beta$ have been given in Theorem 4.6. Obviously, $H(t)$ is nonnegative, and $H(t)=0$ if and only if $u=\bar{u}, v=\bar{v}$, and $w=\bar{w}$. When $(u, v, w)$ is a positive solution of system 
(1.6), $H(t)$ is well posed for all $t \geq 0$ from Theorem 4.5. According to system (1.6), the time derivative of $H(t)$ satisfies

$$
\begin{aligned}
& \frac{d H(t)}{d t}=-\int_{\Omega}\left[\frac{\alpha \bar{u}}{u^{2}}\left(d_{1}+2 \alpha_{11} u\right)|\nabla u|^{2}+\left(d_{2}+2 \alpha_{22} v\right)|\nabla v|^{2}\right. \\
&\left.+\frac{\beta \bar{w}}{w^{2}}\left(d_{3}+\alpha_{31} u+\alpha_{32} v+2 \alpha_{33} w\right)|\nabla w|^{2}+\beta \frac{\alpha_{31} \bar{w}}{w} \nabla u \nabla w+\beta \frac{\alpha_{32} \bar{w}}{w} \nabla v \nabla w\right] d x \\
&-\int_{\Omega}\left\{\alpha\left[\left(\frac{a_{0}}{u}-a_{2}\right)+a_{3}(u+\bar{u})\right](u-\bar{u})^{2}+(v-\bar{v})^{2}+\beta(w-\bar{w})^{2}\right. \\
&\left.-\left(1+\frac{a_{0} \alpha}{u}\right)(u-\bar{u})(v-\bar{v})+(k \alpha-\beta)(u-\bar{u})(w-\bar{w})\right\} d x \\
& \leq- \int_{\Omega}\left[\frac{\alpha \bar{u}}{u^{2}}\left(d_{1}+2 \alpha_{11} u\right)|\nabla u|^{2}+\left(d_{2}+2 \alpha_{22} v\right)|\nabla v|^{2}\right. \\
&\left.+\frac{\beta \bar{w}}{w^{2}}\left(d_{3}+\alpha_{31} u+\alpha_{32} v+2 \alpha_{33} w\right)|\nabla w|^{2}+\beta \frac{\alpha_{31} \bar{w}}{w} \nabla u \nabla w+\beta \frac{\alpha_{32} \bar{w}}{w} \nabla v \nabla w\right] d x \\
&- \int_{\Omega}\left\{\alpha\left[\frac{a_{0}}{u}-\frac{a_{2}\left(M+a_{0} / a_{2}+1 / a_{2}+a_{0}^{2} / 4 a_{2} a_{3} \bar{u}\right)}{u}\right](u-\bar{u})^{2}\right. \\
&\left.-\frac{a_{0} \alpha}{u}(u-\bar{u})(v-\bar{v})-\frac{1}{u}(v-\bar{v})^{2}\right\} d x \\
&- \int_{\Omega}\left[\alpha \frac{a_{3} \bar{u}}{2}(u-\bar{u})^{2}-(u-\bar{u})(v-\bar{v})+(v-\bar{v})^{2}\right] d x \\
&-\int_{\Omega}\left[\alpha \frac{a_{3} \bar{u}}{2}(u-\bar{u})^{2}+(k \alpha-\beta)(u-\bar{u})(w-\bar{w})+\beta(\mathrm{w}-\bar{w})^{2}\right] d x .
\end{aligned}
$$

It is easy to check that the final three integrands on the right side of the above expression are positive definite because of the electing of $\alpha, \beta$, and the sufficient and necessary conditions of the first integrand being positive definite are the following:

$$
\begin{gathered}
4 \alpha \beta \bar{u} \bar{w}\left(d_{1}+2 \alpha_{11} u\right)\left(d_{2}+2 \alpha_{22} v\right)\left(d_{3}+\alpha_{31} u+\alpha_{32} v+2 \alpha_{33} w\right) \\
>\alpha \bar{u}\left(\beta \alpha_{32} \bar{w}\right)^{2}\left(d_{1}+2 \alpha_{11} u\right)+\left(\beta \alpha_{31} \bar{w} u\right)^{2}\left(d_{2}+2 \alpha_{22} v\right) .
\end{gathered}
$$

Noticing that (4.44) is the sufficient conditions of (4.47), so there exists a positive constant $\delta>0$ such that

$$
\begin{aligned}
& \frac{d H(t)}{d t} \leq-\delta \int_{\Omega}\left[(u-\bar{u})^{2}+(v-\bar{v})^{2}+(w-\bar{w})^{2}\right] d x, \\
& \frac{d H(t)}{d t}<0 \quad(u, v, w) \neq(\bar{u}, \bar{v}, \bar{w}) .
\end{aligned}
$$


Similar to the tedious calculations of $d H(t) / d t$, using integration by parts, Hölder inequality, and (4.34), one can verify that $(d / d t) \int_{\Omega}\left[(u-\bar{u})^{2}+(v-\bar{v})^{2}+(w-\bar{w})^{2}\right] d x$ is bounded from above. Thus we have from (4.48) and Lemma 3.2 in Section 3 that

$$
|u(\cdot, t)-\bar{u}|_{2} \longrightarrow 0, \quad|v(\cdot, t)-\bar{v}|_{2} \longrightarrow 0, \quad|w(\cdot, t)-\bar{w}|_{2} \longrightarrow 0 \quad(t \longrightarrow \infty)
$$

In addition, $H(t)$ is decreasing for $t \geq 0$, so we can conclude that the solution $(\bar{u}, \bar{v}, \bar{w})$ is globally asymptotically stable. The proof of Theorem 4.6 is completed.

\section{Acknowledgments}

The work of this author was partially supported by the Natural Science Foundation of Anhui Province Education Department (KJ2009B101) and the NSF of Chizhou College (XK0833) (caguhh@yahoo.com.cn). The work of this author was partially supported by the China National Natural Science Foundation (10871160), the NSF of Gansu Province (096RJZA118), and NWNU-KJCXGC-03-47, 61 Foundations (fusm@nwnu.edu.cn).

\section{References}

[1] E. Kuno, "Mathematical models for predator-prey interaction," Advances in Ecological Research, vol. 16, pp. 252-265, 1987.

[2] J. B. Zheng, Z. X. Yu, and J. T. Sun, "Existence and uniqueness of limit cycle for prey-predator systems with sparse effect," Journal of Biomathematics, vol. 16, no. 2, pp. 156-161, 2001.

[3] C. Shen and B. Q. Shen, "A necessary and sufficient condition of the existence and uniqueness of the limit cycle for a class of prey-predator systems with sparse effect," Journal of Biomathematics, vol. 18, no. 2, pp. 207-210, 2003.

[4] X. Huang, Y. Wang, and L. Zhu, "One and three limit cycles in a cubic predator-prey system," Mathematical Methods in the Applied Sciences, vol. 30, no. 5, pp. 501-511, 2007.

[5] X. Zhang, L. Chen, and A. U. Neumann, "The stage-structured predator-prey model and optimal harvesting policy," Mathematical Biosciences, vol. 168, no. 2, pp. 201-210, 2000.

[6] N. Shigesada, K. Kawasaki, and E. Teramoto, "Spatial segregation of interacting species," Journal of Theoretical Biology, vol. 79, no. 1, pp. 83-99, 1979.

[7] P. Y. H. Pang and M. Wang, "Strategy and stationary pattern in a three-species predator-prey model," Journal of Differential Equations, vol. 200, no. 2, pp. 245-273, 2004.

[8] K. Kuto, "Stability of steady-state solutions to a prey-predator system with cross-diffusion," Journal of Differential Equations, vol. 197, no. 2, pp. 293-314, 2004.

[9] B. Dubey, B. Das, and J. Hussain, "A predator-prey interaction model with self and cross-diffusion," Ecological Modelling, vol. 141, no. 1-3, pp. 67-76, 2001.

[10] Y. Lou, W.-M. Ni, and Y. Wu, "On the global existence of a cross-diffusion system," Discrete and Continuous Dynamical Systems, vol. 4, no. 2, pp. 193-203, 1998.

[11] Y. S. Choi, R. Lui, and Y. Yamada, "Existence of global solutions for the Shigesada-Kawasaki-Teramoto model with strongly coupled cross-diffusion," Discrete and Continuous Dynamical Systems, vol. 10, no. 3, pp. 719-730, 2004.

[12] R. Zhang, L. Guo, and S. M. Fu, "Global behavior for a diffusive predator-prey model with stagestructure and nonlinear density restriction-II: the case in $\mathbb{R}^{1}$," Boundary Value Problems, vol. 2009, Article ID 654539, 19 pages, 2009.

[13] R. Zhang, L. Guo, and S. M. Fu, "Global behavior for a diffusive predator-prey model with stagestructure and nonlinear density restriction-I: the case in $\mathbb{R}^{n}$," Boundary Value Problems, vol. 2009, Article ID 378763, 26 pages, 2009.

[14] J. K. Hale, Ordinary Differential Equations, Robert E. Krieger, Malabar, Fla, USA, 2nd edition, 1980. 
[15] O. A. Ladyzenskaja, V. A. Solonnikov, and N. N. Uralceva, Linear and Quasilinear Partial Differential Equations of Parabolic Type, vol. 23 of Translations of Mathematical Monographs, American Mathematical Society, Providence, RI, USA, 1968.

[16] M. H. Protter and H. F. Weinberger, Maximum Principles in Differential Equations, Springer, New York, NY, USA, 2nd edition, 1984.

[17] M. X. Wang, Nonlinear Partial Differential Equations of Parabolic Type, Science Press, Beijing, China, 1993.

[18] R. M. May, Stability and Complexity in Model Ecosystems, Princeton Univesity Press, Princeton, NJ, USA, 1974.

[19] D. Henry, Geometric Theory of Semilinear Parabolic Equations, vol. 840 of Lecture Notes in Mathematics, Springer, Berlin, Germany, 1993.

[20] M. H. Protter and H. F. Weinberger, Maximum Principles in Differential Equations, Springer, New York, NY, USA, 2nd edition, 1984.

[21] H. H. Cao and S. M. Fu, “Global solutions for a cubic predator-prey cross-diffusion system with stage structure," Mathematics in Practice and Theory, vol. 38, no. 21, pp. 161-177, 2008. 